\title{
Síndrome metabólica em idosos assistidos na atenção terciária à saúde em Curitiba, Paraná: prevalência e associação com saúde, capacidade funcional, estilo de vida e fatores demográficos e socioeconômicos
}

\author{
Metabolic syndrome in elderly assisted in tertiary health care in Curitiba, \\ Paraná, Brazil: prevalence and association with health, functional capacity, \\ life style, and demographic and socioeconomic factors
}

\author{
Adriane Miró Vianna Benke Pereira ${ }^{1} \bowtie$, Irenio Gomes², Carla Helena Augustin Schwanke ${ }^{2}$ \\ ${ }^{1}$ Ambulatório de Saúde do Idoso do Hospital de Clínicas da Universidade Federal do Paraná (HC-UFPR). Curitiba, PR. \\ Programa de Pós-graduação em Gerontologia Biomédica do Instituto de Geriatria e Gerontologia da Pontifícia Universidade Católica
}

do Rio Grande do Sul (PUCRS). Porto Alegre, RS.

\section{RESUMO}

Objetivos: Avaliar a prevalência de síndrome metabólica em idosos assistidos na atenção terciária à saúde e a associação entre síndrome metabólica e saúde, capacidade funcional, estilo de vida e fatores demográficos e socioeconômicos.

Métodos: Foi realizado um estudo transversal envolvendo idosos atendidos em um ambulatório de Clínica Médica de um hospital universitário localizado em Curitiba, capital do Paraná. A síndrome metabólica foi definida pelos critérios do National Cholesterol Education Program Adult Treatment Panel III (NCEP-ATPIII). As variáveis avaliadas foram: síndrome metabólica e seus componentes; fatores demográficos e socioeconômicos (faixa etária, sexo, escolaridade, renda familiar); estilo de vida (tabagismo, etilismo, adesão à dieta, atividade física); perfil de saúde (doenças crônicas não transmissíveis, medicamentos de uso contínuo); e capacidade funcional, determinada através das escalas de Katz e de Lawton.

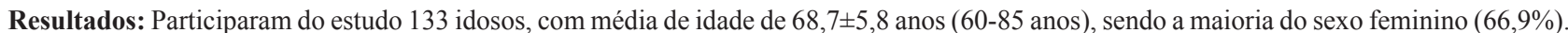
A prevalência de síndrome metabólica foi de $63,9 \%$, sendo $71,8 \%$ entre mulheres e $28,2 \%$ entre os homens ( $\mathrm{p}=0,16)$. Síndrome metabólica foi mais frequente nos indivíduos entre 60-69 anos em comparação com os acima dessa idade $(64,7 \%$ versus $35,3 \%$; $\mathrm{p}=0,03)$. Também houve associação de síndrome metabólica com relato de cinco ou mais doenças crônicas não transmissíveis $(60,0 \%$ versus $29,2 \%, \mathrm{p}<0,001)$ e com uso de sete ou mais medicamentos contínuos $(36,5 \%$ versus $1,6 \%, \mathrm{p}<0,001)$. Obesidade abdominal foi significativamente mais frequente entre as mulheres $(98,4 \%$ versus $66,7 \% ; \mathrm{p}=0,001)$ e hiperglicemia mais frequente entre os homens $(95,8 \%$ versus $75,4 \% ; \mathrm{p}=0,03)$. Apenas $7,1 \%$ dos idosos com síndrome metabólica eram considerados dependentes.

Conclusões: A prevalência de síndrome metabólica foi elevada nesta amostra de idosos assistidos na atenção terciária à saúde. A distribuição de dois dos cinco componentes da síndrome metabólica foi diferenciada entre os sexos (obesidade central mais frequente em mulheres e hiperglicemia em homens). Idosos com síndrome metabólica tinham mais frequentemente menos de 70 anos, relatavam maior número de doenças crônicas e usavam maior número de medicações contínuas. Não se observou associação de síndrome metabólica com sexo, fatores socioeconômicos, estilo de vida e capacidade funcional.

DESCRITORES: síndrome X metabólica/epidemiologia; ambulatório hospitalar; idoso; atividades cotidianas.

\section{ABSTRACT}

Aims: To evaluate the prevalence of metabolic syndrome in elderly assisted in tertiary health care and the association between metabolic syndrome and health, functional capacity, life style, and demographic and socioeconomic factors.

Methods: A cross-sectional study was conducted involving elderly assisted in an outpatient medical clinic of a university hospital in Curitiba, capital of Paraná State, Brazil. Metabolic syndrome was defined according to the National Cholesterol Education Program Adult Treatment Panel III (NCEP-ATPIII). The variables evaluated were: metabolic syndrome and its components; demographic and socioeconomic factors (age, sex, education, family income); lifestyle (smoking, alcohol use, adherence to diet, physical activity); health profile (non-communicable diseases, continued use of medicines); and functional capacity, determined by Katz and Lawton scales.

Results: The study included 133 elderly, with a mean age of $68.7 \pm 5.8$ years $(60-85$ years), most of them women (66.9\%). The prevalence of metabolic syndrome was $63.9 \%$, being $71.8 \%$ in women and $28.2 \%$ in men $(p=0.16)$. Metabolic syndrome was more frequent in individuals aged 60-69 years compared with those above that age $(64.7 \%$ versus $35.3 \% ; \mathrm{p}=0.03)$. There was also association of metabolic syndrome with reporting five or more chronic diseases $(60.0 \%$ versus $29.2 \%, \mathrm{p}<0.001)$ and with using seven or more continuous medicines $(36.5 \%$ versus $1.6 \%, \mathrm{p}<0.001)$. Abdominal obesity was more frequent among women $(98.4 \%$ versus $66.7 \% ; \mathrm{p}=0.001)$ and hyperglycemia was more frequent among men $(95.8 \%$ versus $75.4 \% ; \mathrm{p}=0.03)$. Only $7.1 \%$ of the elderly with metabolic syndrome were considered dependent.

Conclusions: Prevalence of metabolic syndrome was high in this sample of elderly assisted in tertiary health care setting. The distribution of two of the five components of metabolic syndrome was different between sexes (central obesity more frequent in women and hyperglycemia more frequent in men). Elderly people with metabolic syndrome were more often less than 70 years old, reported more chronic diseases and used more continuous medicines. There was no association of metabolic syndrome with sex, socioeconomic factors, lifestyle or functional capacity. KEY WORDS: metabolic syndrome X; hospital outpatient clinics; elderly; activities of daily living. 
Abreviaturas: SM, síndrome metabólica; HC-UFPR, Hospital de Clínicas da Universidade Federal do Paraná; HDL-c, colesterol ligado à lipoproteína de alta densidade; NCEP-ATPIII, National Cholesterol Education Program-Adult Treatment Panel; PA, pressão arterial; HAS, hipertensão arterial sistêmica; CA, circunferência abdominal; DCNT, doenças crônicas não transmissíveis.

\section{INTRODUÇÃO}

A síndrome metabólica ( $\mathrm{SM}$ ) é um transtorno complexo representado por um conjunto de fatores de risco cardiovascular usualmente relacionados à adiposidade central e à resistência à insulina [1]. Seu diagnóstico é feito pela constatação da presença, em um mesmo indivíduo, de obesidade central, dislipidemia, hiperglicemia e hipertensão arterial sistêmica (HAS) [2]. Frequentemente a SM associase a outras condições médicas, especialmente estados pró-trombótico e pró-inflamatório, esteatose hepática, cálculos de colesterol, apneia do sono, gota, depressão, doenças musculoesqueléticas e síndrome dos ovários policísticos [3]. Além dos desfechos cardiovasculares, entre idosos tem sido demonstrada associação de SM com condições de extrema importância no envelhecimento, como declínio da mobilidade, incapacidade, fragilidade, sarcopenia e declínio cognitivo [4-9].

Com o rápido envelhecimento populacional observado mundialmente, a tendência é de que essas situações se tornem mais frequentes na prática clínica, requerendo o desenvolvimento de estratégias capazes de prevenir ou retardar seu surgimento. É, portanto, imperativo conhecer os fatores de risco a que estão expostos os idosos [10].

O objetivo do presente estudo foi avaliar a prevalência da SM em idosos assistidos em um ambulatório localizado em um hospital universitário e analisar a associação entre SM e variáveis demográficas, socioeconômicas e de saúde, hábitos de vida e capacidade funcional.

\section{MÉTODOS}

\section{Delineamento e local do estudo}

Trata-se de um estudo transversal, descritivo e analítico, integrante do projeto "Prevalência de síndrome metabólica em idosos atendidos em um ambulatório de clínica médica de hospital universitário do Sul do Brasil e associação com declínio cognitivo" [10]. O estudo foi realizado no Ambulatório de Clínica Médica do Hospital de Clínicas da Universidade Federal do
Paraná (HC-UFPR), localizado na cidade de Curitiba, capital do estado do Paraná, no período compreendido entre março e outubro de 2010.

\section{População e amostra}

O cálculo da amostra foi feito considerando-se o escore do Mini Exame do Estado Mental (MEEM) em uma população de idosos semelhante à estudada neste projeto, cujo escore médio foi de aproximadamente 25 com desvio padrão de aproximadamente 4 [11]. Considerou-se que pelo menos dois seria uma diferença clinicamente importante entre os dois grupos de comparação. Considerando ainda proporção de 1,5:1 entre os grupos (prevalência de SM de aproximadamente 60\%) [12], foi estimada amostra de 135 idosos. Este cálculo foi feito utilizando o programa "samples.exe" do pacote estatístico PEPI versão 4, considerando um poder de $90 \%$ e um erro alfa de 0,05 .

Participaram do estudo indivíduos com idade igual ou maior que 60 anos, convidados aleatoriamente a compor a amostra no dia de suas consultas de rotina. Foram excluídos idosos com deficiência auditiva, visual ou motora grave que pudesse prejudicar o desempenho nas avaliações propostas.

\section{Variáveis e métodos de mensuração}

Foram avaliadas variáveis relativas ao diagnóstico de SM: pressão arterial (PA), circunferência abdominal, colesterol ligado a lipoproteína de alta densidade (HDL-c), glicose de jejum e triglicerídios; variáveis demográficas: sexo e idade; variáveis socioeconômicas: escolaridade e renda familiar; variáveis de saúde: doenças crônicas não transmissíveis (DCNT) e medicamentos de uso contínuo; hábitos de vida: etilismo, tabagismo, alimentação e atividade física; e capacidade funcional.

A classificação para SM foi baseada no critério diagnóstico proposto pelo Third Adult Treatment Panel of the National Cholesterol Education Program. (NCEP-ATPIII) [2], que considera portadores de SM indivíduos que preencham três dos cinco seguintes parâmetros: circunferência abdominal aumentada ( $\geq 88 \mathrm{~cm}$ para mulheres e $\geq 102 \mathrm{~cm}$ para homens); triglicerídeos aumentados $(\geq 150 \mathrm{mg} / \mathrm{dL})$; HDL-c reduzido $(<40 \mathrm{mg} / \mathrm{dL}$ para homens e $<50 \mathrm{mg} / \mathrm{dL}$ para mulheres), glicemia de jejum elevada ( $\geq 110 \mathrm{mg} / \mathrm{dL})$; $\mathrm{PA} \geq 135 / 80 \mathrm{mmHg}$; uso de medicações hipolipemiantes, hipoglicemiantes ou anti-hipertensivas.

A circunferência abdominal (CA) foi aferida com fita métrica inelástica no ponto médio entre o 
rebordo costal e a crista ilíaca, com o indivíduo em pé, obedecidos os pontos de corte para homens $\geq 102$ $\mathrm{cm}$ e para mulheres $\geq 88 \mathrm{~cm}$, conforme recomendações da I Diretriz Brasileira de Diagnóstico e Tratamento da Síndrome Metabólica [1].

Seguindo orientações contidas nas VI Diretrizes Brasileiras de Hipertensão Arterial [13], a PA foi aferida no braço, utilizando-se esfigmomanômetro de mercúrio calibrado e braçadeira adequada para obesos quando necessário.

Para avaliação do perfil bioquímico os participantes foram orientados a manter 12 horas de jejum para a coleta de sangue e os exames foram realizados no Laboratório de Análises Clínicas do HC-UFPR, utilizando a metodologia de Colorimetria pelo equipamento Architect CI 3.200 (Abott, Wiesbadem, Alemanha).

A classificação da capacidade funcional foi feita através das Escalas de Katz [14] para avaliação das atividades de vida diária e Escala de Lawton [14] para as atividades instrumentais de vida diária. Os participantes foram classificados inicialmente em independentes, parcialmente dependentes e dependentes para cada escala. Os participantes classificados como dependentes e parcialmente dependentes foram reunidos em um único grupo, denominado dependente.

Em relação à adesão à alimentação adequada, foram considerados como adeptos os participantes que relataram fazer uso de qualquer tipo de dieta alimentar orientada, quer fosse com finalidades terapêuticas ou preventivas.

Foram considerados fisicamente ativos os idosos que informassem a prática rotineira de no mínimo 30 minutos de atividade física em pelo menos três dias da semana.

Foram classificados como tabagistas os participantes que, na época da pesquisa fumavam cigarros diariamente; não tabagistas aqueles que nunca fumaram cigarros; e ex-tabagistas aqueles que informaram ter fumado cigarros habitualmente no passado. Para as análises estatísticas, participantes tabagistas e extabagistas foram agrupados e classificados todos como tabagistas.

Foram classificados como etilistas habituais os participantes que na época da pesquisa ingeriam bebidas alcoólicas diariamente; ex-etilistas aqueles que informaram ter consumido bebidas alcoólicas regularmente no passado; não etilistas aqueles que nunca ingeriram bebidas alcoólicas; e etilistas eventuais aqueles que relataram consumir apenas eventualmente quantidades pequenas de bebidas alcoólicas. Para a análise estatística os participantes que se declararam etilistas habituais e ex-etilistas foram classificados como etilistas. Os participantes que declararam nunca ter ingerido bebidas alcoólicas ou consumido eventualmente pequenas quantidades foram classificados como não etilistas.

Para coleta de informações socioeconômicas e condições de saúde foi utilizado questionário elaborado especificamente para o presente estudo. Testado em experiência piloto, incluiu informações sobre sexo, idade, escolaridade, renda familiar, DCNT autorrelatadas e medicamentos em uso contínuo.

\section{Análise estatística}

Os dados foram armazenados em banco de dados desenvolvido para o projeto no Microsoft Access 2007 e analisados pelo programa estatístico SPSS versão 17.0. A apresentação dos resultados ocorreu pela estatística descritiva através das medidas de tendência central (média e mediana) e de variabilidade (desvio padrão e amplitude interquartis), bem como, distribuição absoluta e relativa. A simetria das distribuições contínuas foi avaliada pelo teste de KolmogorovSmirnov. Para a comparação de proporções entre as categorias de uma mesma variável (análise univariada), foi utilizado o teste qui-quadrado de Pearson, levando em consideração a distribuição teórica de homogeneidade entre as categorias comparadas. Para a análise bivariada entre variáveis qualitativas foram utilizados o teste qui-quadrado de Pearson e a correção de continuidade. Foi utilizada a análise pelos resíduos ajustados, em que os valores negativos indicam uma frequência real inferior à esperada e os valores positivos uma frequência real superior à esperada. As células cujos resíduos ajustados assumem valores iguais ou acima de 1,96 em valor absoluto, contribuem significativamente para a relação de dependência entre variáveis comparadas [15]. Nas tabelas de contingência em que pelo menos $25 \%$ dos valores das caselas apresentassem frequência esperada menor do que 5, foi utilizado o teste exato de Fisher, sendo que, nas situações onde pelo menos uma variável tivesse característica politômica, foi utilizada a simulação de Monte Carlo. Para as variáveis contínuas, quando a comparação ocorreu entre dois grupos independentes, foi aplicado o teste $t$ de Student.

\section{Aspectos éticos}

O projeto foi aprovado pela Comissão Científica do Instituto de Geriatria e Gerontologia da Pontifícia Universidade Católica do Rio Grande do Sul (PUCRS) 
e pelo Comitê de Ética em Pesquisa da PUCRS. O desenvolvimento do estudo nas dependências do HC-UFPR foi autorizado pela direção clínica desse hospital. Todos os pesquisadores e participantes assinaram duas cópias do termo de consentimento livre e esclarecido. Foram seguidos todos os preceitos éticos descritos na Resolução 453/2012 do Conselho Nacional de Saúde do Ministério da Saúde do Brasil.

\section{RESULTADOS}

Cento e trinta e cinco idosos atenderam aos critérios de inclusão, tendo sido excluídos dois por não terem realizado os exames laboratoriais necessários. Assim, a amostra final foi composta por 133 idosos, sendo $89(67 \%)$ mulheres e $44(33 \%)$ homens. A média de idade foi de $68,7 \pm 5,8$ anos, sendo 60 anos a idade mínima e 85 anos a idade máxima. A prevalência de SM foi de $63,9 \%(n=85)$.

A média da idade dos idosos com SM mostrou-se significativamente menor que a dos participantes sem SM $(67,6 \pm 5,6$ anos vs. $70,5 \pm 5,9$ anos; $p<0,01)$. Os demais fatores demográficos e socioeconômicos da amostra estão descritas na Tabela 1. Foi detectada associação da SM apenas com faixa etária, sendo que a prevalência de SM foi significativamente maior entre os indivíduos com idade entre 60 e 69 anos em relação aos indivíduos mais velhos. Em relação ao sexo, escolaridade e renda familiar, não se observaram diferenças estatisticamente significativas.

Comparando a distribuição dos componentes da SM entre os sexos, verificou-se que a frequência de circunferência abdominal aumentada foi significativamente maior no sexo feminino $(p<0,001)$. Já a frequência de hiperglicemia de jejum foi significativamente maior no sexo masculino $(p=0,032)$. Não houve diferenças significativas entre os sexos em relação aos demais componentes da SM (Tabela 2).

A caracterização da amostra quanto a fatores de saúde e de estilo de vida encontra-se descrita na Tabela 3. Houve associação significativa da SM com o número de DCNT relatadas e uso de medicamentos contínuos. A associação configurou-se com o relato de cinco ou mais doenças e com o uso de sete ou mais medicamentos. No caso dos medicamentos, observouse ainda associação linear $(\mathrm{p}<0,001)$, indicando que, quanto maior o número de medicamentos maior a chance de apresentar a SM. Não houve associação significativa com funcionalidade, atividade física, adesão a dieta, tabagismo ou etilismo (Tabela 3).

Tabela 1. Características demográficas e socioeconômicas de idosos atendidos no Ambulatório de Clínica Médica do Hospital de Clínicas da Universidade Federal do Paraná (HC-UFPR) na amostra total e nos indivíduos com e sem síndrome metabólica. 2010.

\begin{tabular}{|c|c|c|c|c|}
\hline \multirow[b]{2}{*}{ Variáveis } & \multirow[b]{2}{*}{$\begin{array}{l}\text { Amostra total } \\
\qquad \begin{array}{c}(n=133) \\
n(\%)\end{array}\end{array}$} & \multicolumn{2}{|c|}{ Síndrome metabólica } & \multirow[b]{2}{*}{$p^{\S}$} \\
\hline & & $\begin{array}{c}\underset{\text { Sim }}{(\mathbf{n}=85)} \\
\mathbf{n}(\%)^{*}\end{array}$ & $\begin{array}{c}\text { Não } \\
(\mathrm{n}=48) \\
\mathrm{n}(\%)^{*}\end{array}$ & \\
\hline Sexo & & & & 0,165 \\
\hline Feminino & $89(66,9)$ & $61(71,8)$ & $28(58,3)$ & \\
\hline Masculino & $44(33,1)$ & $24(28,2)$ & $20(41,7)$ & \\
\hline Faixa etária & & & & 0,03 \\
\hline 60 a 69 anos & $77(57,9)$ & $55(64,7)$ & $22(45,8)$ & \\
\hline 70 anos ou mais & $56(42,1)$ & $30(35,3)$ & $26(54,2)$ & \\
\hline Escolaridade & & & & 0,092 \\
\hline Analfabetos & $18(13,5)$ & $14(16,5)$ & $4(8,3)$ & \\
\hline Escolarizados & $115(86,5)$ & $71(83,5)$ & $44(91,7)$ & \\
\hline Renda familiar ${ }^{\dagger}$ (salário mínimo) & & & & $0,515^{\ddagger}$ \\
\hline De $1 / 2$ a 1 & $23(20,4)$ & $12(16,2)$ & $11(28,2)$ & \\
\hline Mais de 1 a 2 & $35(31,0)$ & $25(33,8)$ & $10(25,6)$ & \\
\hline Mais de 2 a 3 & $27(23,9)$ & $17(23,0)$ & $10(25,6)$ & \\
\hline Mais de 3 a 5 & $20(17,7)$ & $15(20,3)$ & $5(12,8)$ & \\
\hline Mais de 5 & $8(7,1)$ & $5(6,8)$ & $3(7,7)$ & \\
\hline
\end{tabular}

* Percentuais obtidos com base no total de cada categoria da SM.

+ Não se aplica/não respondeu $=20$ (15\%).

$\S$ Teste qui-quadrado de Pearson com correção de continuidade ou teste exato de Fisher por simulação de Monte Carlo.

₹ Teste qui-quadrado de Pearson. 
Tabela 2. Positividade do diagnóstico dos componentes da síndrome metabólica observada em idosos atendidos no Ambulatório de Clínica Médica do Hospital de Clínicas da Universidade Federal do Paraná (HC-UFPR) na amostra total e nos sexos feminino e masculino. 2010.

\begin{tabular}{|c|c|c|c|c|}
\hline \multirow[b]{2}{*}{$\begin{array}{c}\text { Componentes da síndrome } \\
\text { metabólica }\end{array}$} & \multirow[b]{2}{*}{$\begin{array}{c}\text { Amostra total } \\
\text { n (\%) }\end{array}$} & \multicolumn{2}{|c|}{ Sexo } & \multirow[b]{2}{*}{$p \S$} \\
\hline & & $\begin{array}{c}\text { Feminino } \\
(\mathrm{n}=89) \\
\mathrm{n}(\%)\end{array}$ & $\begin{array}{c}\text { Masculino } \\
(\mathrm{n}=44) \\
\mathrm{n}(\%)\end{array}$ & \\
\hline \multicolumn{5}{|l|}{ Amostra total $(n=133)^{*}$} \\
\hline PA elevada & $119(89,5)$ & $80(89,9)$ & $39(88,6)$ & 0,926 \\
\hline Obesidade abdominal & $89(66,9)$ & $68(76,4)$ & $21(47,7)$ & 0,002 \\
\hline Glicose de jejum elevada & $94(70,7)$ & $59(66,3)$ & $35(79,5)$ & 0,168 \\
\hline HDL-c reduzido & $81(60,9)$ & $55(61,8)$ & $26(59,1)$ & 0,856 \\
\hline Triglicerídeos elevados & $52(39,1)$ & $34(38,2)$ & $18(40,9)$ & 0,763 \\
\hline Idosos com SM $(n=85)^{\dagger}$ & $85(63,9)$ & $61(71,8)$ & $24(28,2)$ & \\
\hline PA elevada & $81(95,3)$ & $58(95,1)$ & $23(95,8)$ & 0,679 \\
\hline Obesidade abdominal & $76(85,4)$ & $60(98,4)$ & $16(66,7)$ & 0,001 \\
\hline Glicose de jejum elevada & $69(73,4)$ & $46(75,4)$ & $23(95,8)$ & 0,032 \\
\hline HDL-c reduzido & $65(76,5)$ & $32(52,5)$ & $15(62,5)$ & 0,472 \\
\hline Idosos sem SM $(n=48)^{\ddagger}$ & $48(36,1)$ & $28(58,3)$ & $20(41,7)$ & \\
\hline PA elevada & $38(79,2)$ & $22(78,6)$ & $16(80,0)$ & 0,882 \\
\hline Obesidade abdominal & $37(77,1)$ & $21(75,0)$ & $16(80,0)$ & 1,000 \\
\hline Glicose de jejum elevada & $25(52,1)$ & $13(46,4)$ & $12(60,0)$ & 0,394 \\
\hline HDL-c reduzido & $16(33,3)$ & $9(32,1)$ & $7(35,0)$ & 0,155 \\
\hline Triglicerídeos aumentados & $5(10,4)$ & $02(7,1)$ & $3(15,0)$ & 0,636 \\
\hline
\end{tabular}

PA, pressão arterial; HDL-c, colesterol ligado à lipoproteína de alta densidade; SM, síndrome metabólica.

* Percentuais obtidos com base no total da amostra ou sobre o total de casos de cada sexo; † Percentuais obtidos com base no total de pacientes com SM, bem como sobre o total de cada sexo que apresentou SM; ₹ Percentuais obtidos com base no total de pacientes sem SM, bem como sobre o total de cada sexo que não apresentou SM; § Teste qui-quadrado de Pearson com correção de continuidade ou teste exato de Fisher por simulação de Monte Carlo.

Tabela 3. Distribuição absoluta e relativa de condições de saúde, capacidade funcional e estilo de vida de idosos atendidos no Ambulatório de Clínica Médica do Hospital de Clínicas da Universidade Federal do Paraná (HC-UFPR), segundo a presença ou não de síndrome metabólica (n=133). 2010.

\begin{tabular}{|c|c|c|c|c|}
\hline \multirow[b]{2}{*}{ Variáveis } & \multirow[b]{2}{*}{$\begin{array}{c}\text { Amostra total } \\
(n=133) \\
n(\%)\end{array}$} & \multicolumn{2}{|c|}{ Síndrome metabólica } & \multirow[b]{2}{*}{$\mathrm{p} \S$} \\
\hline & & $\begin{array}{c}\text { Sim } \\
(n=85) \\
n(\%) *\end{array}$ & $\begin{array}{c}\text { Não } \\
(n=48) \\
n(\%)^{*}\end{array}$ & \\
\hline Capacidade funcional & & & & 0,769 \\
\hline Dependente & $08(6,0)$ & $06(7,1)$ & $02(4,2)$ & \\
\hline Independente & $125(94,0)$ & $79(92,9)$ & $46(95,8)$ & \\
\hline Número de doenças & & & & $<0,001$ \\
\hline 1 a 2 & $21(15,8)$ & $05(5,9)$ & $16(33,3)$ & \\
\hline 3 a 4 & $47(35,3)$ & $29(34,1)$ & $18(37,5)$ & \\
\hline 5 a 6 & $52(39,1)$ & $39(45,9)$ & $13(27,1)$ & \\
\hline 7 ou mais & $13(9,8)$ & $12(14,1)$ & $01(2,1)$ & \\
\hline Número de medicamentos & & & & $<0,001$ \\
\hline 1 a 2 & $22(16,5)$ & $04(4,7)$ & $18(43,8)$ & \\
\hline 3 a 4 & $36(27,1)$ & $24(28,2)$ & $12(27,3)$ & \\
\hline 5 a 6 & $38(28,6)$ & $26(30,6)$ & $12(27,3)$ & \\
\hline 7 ou mais & $37(27,8)$ & $31(36,5)$ & $06(1,6)$ & \\
\hline Tabagismo & & & & 1,000 \\
\hline Sim & $56(42,1)$ & $36(42,4)$ & $20(41,7)$ & \\
\hline Não & $77(57,9)$ & $49(57,6)$ & $28(58,3)$ & \\
\hline Etilismo & & & & 0,998 \\
\hline $\operatorname{Sim}$ & $18(13,6)$ & $11(12,9)$ & $07(85,4)$ & \\
\hline Não & $115(86,4)$ & $74(87,1)$ & $41(85,4)$ & \\
\hline Adesão à dieta & & & & 0,796 \\
\hline Não & $98(73,7)$ & $62(72,9)$ & $36(75,0)$ & \\
\hline Sim & $35(26,3)$ & $23(27,1)$ & $12(25,0)$ & \\
\hline Atividade física & & & & 0,697 \\
\hline Não & $83(62,4)$ & $52(61,2)$ & $31(64,6)$ & \\
\hline Sim & $50(37,6)$ & $33(38,8)$ & $17(35,4)$ & \\
\hline
\end{tabular}

* Percentuais obtidos com base no total de cada categoria da SM.

§ Teste qui-quadrado de Pearson com correção de continuidade ou teste exato de Fisher por simulação de Monte Carlo. 


\section{DISCUSSÃO}

No presente estudo, a SM foi definida pelo critério proposto pelo NCEP-ATPIII [2], por ser o critério recomendado pela I Diretriz Brasileira de Diagnóstico e Tratamento da Síndrome Metabólica [1] para uso no Brasil, por ser citado como o melhor preditor de desfecho cardiovascular em idosos [16] e por ser o critério mais usado nos estudos de prevalência de $\mathrm{SM}$ em idosos. Os estudos de prevalência de SM têm mostrado resultados variáveis, dependentes do critério utilizado para sua definição e das características da população investigada. Assim, foram selecionados para comparação com nossos resultados, estudos de prevalência de SM em idosos que também usaram o critério proposto pelo NCEP-ATPIII [2].

A prevalência de SM encontrada nesta pesquisa foi muito elevada, maior do que a verificada em diversos estudos internacionais [7,17-23]. Com exceção de um estudo francês [19], que considerou idosos atendidos na atenção primária em saúde de Paris, os estudos referenciados recrutaram seus participantes na comunidade. A maior prevalência de SM observada na amostra do presente estudo provavelmente deve-se ao fato de se tratar de um grupo de idosos atendidos em ambulatório de atenção terciária, de referência para a região, para onde convergem pacientes de alta complexidade.

A porcentagem de mulheres com SM observada neste estudo foi maior do que a observada entre os homens, entretanto a diferença não foi significativa, em razão de haver uma proporção muito maior de mulheres na amostra. No entanto, maior prevalência de SM em mulheres tem sido observada em outros estudos, tanto nacionais quanto internacionais $[16,18$, 20-22]. No Brasil, conforme revisão da literatura realizada por Lopes [24], em todos os estudos que apresentaram a prevalência da SM de acordo com o gênero, as mulheres apresentaram prevalência superior a dos homens, informação que também se repete na comparação com outras pesquisas brasileiras realizadas entre idosos [25-31].

Quando comparada à de estudos brasileiros [25-31], a prevalência de SM também foi maior na presente amostra. $\mathrm{O}$ ambiente de pesquisa que mais se aproximou do presente estudo, ou seja, um ambulatório de atenção terciária, foi o estudado por Cabrera et al. [25], em que foram investigadas mulheres idosas atendidas em um ambulatório de geriatria de um hospital universitário paranaense. Naquele estudo verificouse prevalência de SM de 39,9\%, muito inferior à das mulheres participantes desta amostra $(71,8 \%)$. Esta diferença pode ser explicada em parte pelo ponto de corte usado por Cabrera et al. [25] para a definição de HAS, que foi de $140 / 90 \mathrm{mmHg}$. Outra diferença foi que Cabrera não considerou para o diagnóstico de SM a dislipidemia em tratamento. Ao usar o ponto de corte indicado pelo NCEP-ATPIII [2], de 135/80 $\mathrm{mmHg}$, provavelmente classificamos maior número de participantes como portadores de SM. Usando o ponto de corte de 140/90 $\mathrm{mmHg}$, a prevalência de SM em nosso estudo teria sido de $55,6 \%$, um pouco menor do que a observada com o ponto de corte de 130/85. Embora a HAS seja considerada como o principal fator de risco cardiovascular entre idosos [32] e o ponto de corte para PA recomendado para esta população seja maior do que o sugerido para adultos [32,33], nenhuma das definições de SM considera aspectos particulares dos idosos entre seus critérios, questão que deve receber atenção. Considerando-se que a PA alterada foi o componente da SM mais frequente na presente amostra, estas observações tornam-se ainda mais relevantes, pois ao aplicar parâmetros que são considerados normais para adultos, mas não para idosos, corremos o risco de superestimar a prevalência da condição pesquisada.

Como em outros estudos [20,25,29], entre as mulheres portadoras da SM, a circunferência abdominal aumentada foi o componente mais frequentemente observado na presente amostra. Durante o envelhecimento, a quantidade de gordura permanece estável, porém ocorrem alterações na distribuição da gordura corporal, com aumento da gordura abdominal [34]. Rosemberg [35], em sua dissertação de mestrado, que envolveu a avaliação de 518 idosos atendidos na Estratégia Saúde da Família do Município de Porto Alegre/RS, verificou que o ponto de corte da circunferência abdominal para SM era $\geq 94,8 \mathrm{~cm}$ para homens e $\geq 91,7 \mathrm{~cm}$ para mulheres. Ou seja, o ponto de corte foi menor para os homens e maior para as mulheres em relação àqueles propostos pelo NCEP-ATPIII revisado ( $\geq 102 \mathrm{~cm}$ para homens e $\geq 88 \mathrm{~cm}$ para mulheres). Como em relação aos níveis de PA, talvez seja necessário reconsiderar parâmetros de circunferência abdominal mais adequados à população idosa.

O aumento da prevalência da SM com o avançar da idade é fato frequentemente citado na literatura, mas é importante observar que em vários desses estudos [36-41] as faixas etárias analisadas incluíram os idosos como um grupo homogêneo, com idades a partir de 60 ou 65 anos, e geralmente comparados a faixas etárias inferiores. Ao analisar os subgrupos etários como no presente estudo (idosos mais jovens com 
idosos mais velhos), outros pesquisadores também observaram associação da SM aos subgrupos mais jovens $[30,40,41]$. Tem sido sugerido que indivíduos longevos parecem ser capazes de retardar ou mesmo evitar o surgimento de várias das doenças relacionadas ao envelhecimento, como diabetes mellitus tipo 2 e doenças cardiovasculares, talvez por serem portadores de traços genéticos e fisiológicos que de alguma forma os protejam e favoreçam a maior longevidade $[42,43]$.

A associação da SM ao relato da existência de cinco ou mais doenças e ao uso de sete ou mais medicamentos, verificada no presente estudo, pressupõe maiores gastos com saúde, fato corroborado pelo estudo de Curtis et al. [44], em que se verificou aumento dos custos com saúde em idosos portadores de SM.

A associação da SM ao declínio funcional é um aspecto controverso na literatura e o número de pesquisas relacionadas ao tema ainda é limitado [5]. Entre quatro estudos que avaliaram associação da SM ao declínio funcional considerando as atividades de vida diária $[5,26,45,46]$, dois $[45,46]$ não conseguiram demonstrar essa relação, assim como ocorreu no presente estudo. Outros quatro estudos [4,47-49] analisaram associação entre SM e a funcionalidade do ponto de vista da mobilidade. Em três deles [4,48, 49], de características longitudinais, a associação foi identificada, enquanto que um estudo transversal brasileiro [50] não observou a relação. Ainda, estudo retrospectivo de participantes do National Health and Nutrition Examination Survey 1999-2002 [50] registrou associação da SM com incapacidade tanto do ponto de vista das atividades diárias como da mobilidade, definindo obesidade abdominal e aumento de triglicerídeos como preditores de dependência funcional.

Diferenças na população estudada e questões metodológicas podem ser responsáveis pelos resultados divergentes encontrados em relação à funcionalidade. Diante da importância da capacidade funcional e física para a manutenção da independência no envelhecimento, consideramos necessárias análises posteriores desta amostra de idosos, com observação prospectiva e análise dos fatores de confusão envolvidos na relação entre SM e capacidade funcional.

Uma questão que emerge deste estudo é a necessidade de discutir o desenvolvimento de um critério diagnóstico de SM específico para uso em idosos, que considere suas peculiaridades. Os critérios atuais que definem a SM aplicam os mesmos pontos de corte de seus componentes para todos os indivíduos, sem diferença entre as idades [51]. Wu et al. [51] também sugerem a necessidade de se reconsiderar os pontos de corte para indivíduos de diferentes grupos etários.

Este estudo teve algumas limitações. O menor número de participantes do sexo masculino prejudicou a avaliação da prevalência da SM por sexos e o pequeno número de participantes com idades superiores a 80 anos restringiu as análises referentes aos subgrupos etários. Além disto, com respeito à avaliação dos hábitos de vida, foram observados aspectos da prática clínica diária em lugar de roteiros formais. Essa opção foi feita tendo em vista o tempo necessário para a aplicação dos instrumentos, uma vez que a avaliação geriátrica é usualmente mais extensa do que a avaliação clínica usual. Assim, os resultados relativos aos hábitos de vida dos participantes devem ser considerados com cautela.

Apesar de sua característica transversal, este estudo identificou que a prevalência de $\mathrm{SM}$ em idosos em atendimento ambulatorial terciário foi elevada, que a distribuição dos componentes da SM foi diferenciada entre os sexos e que os pacientes com SM eram mais frequentemente idosos mais jovens (até 69 anos), relataram um número maior de DCNT (cinco ou mais) e usavam número maior de medicamentos contínuos (sete ou mais). Contudo, não se observou associação de SM com sexo, fatores socioeconômicos, estilo de vida e capacidade funcional. Estes resultados apontam para a necessidade da adoção de medidas de promoção e prevenção de saúde em todos os ciclos de vida, para que os indivíduos possam alcançar idades avançadas em melhores condições de saúde e de qualidade de vida.

\section{NOTA}

Declaração de conflitos de interesse

Os autores declaram não haver conflitos de interesse relevantes ao conteúdo deste estudo.

\section{REFERÊNCIAS}

1. Sociedade Brasileira de Cardiologia. I Diretriz Brasileira de Diagnóstico e Tratamento da Síndrome Metabólica. Arq Bras Cardiol. 2005;84 supl.1:3-28. http://dx.doi.org/10.1590/S0066-782X2005000700001 
2. Executive Summary of the Third Report of the National Cholesterol Education Program (NCEP) Expert Panel on Detection, Evaluation, and Treatment of High Blood Cholesterol in Adults (Adult treatment Panel III). JAMA. 2001;285:2486-97. http://dx.doi.org/10.1001/ jama.285.19.2486

3. Scott M. Grundy MF. Metabolic syndrome pandemic. Arterioscler Thromb Vasc Biol. 2008;28:629-36. http://dx.doi.org/10.1161/ ATVBAHA.107.151092

4. Blazer DG, Hybels CF, Fillenbaum GG. Metabolic syndrome predicts mobility decline in a community-based sample of older adults. JAGS. 2006;54:502-6. http://dx.doi.org/10.1111/j.1532-5415.2005.00607.x

5. Carriere I, Peres K, Ancelin ML, Veronique G, Berr C, Barberger-Gateau P, Bouillon K, Kivimaki M, Karen Ritchie K, Akbaraly T. Metabolic syndrome and disability: findings from the Prospective Three-City Study. J Gerontol A Biol Sci Med Sci. 2014;69:79-86. http:/ dx.doi.org/10.1093/gerona/glt101

6. Viscogliosi G. The Metabolic Syndrome: A risk factor for the frailty syndrome? JAMDA. 2016;17:364-9. http://dx.doi.org/10.1016/j. jamda.2016.01.005

7. Ishii S, Tanaka T, Akishita M, Ouchi Y, Tuji T, Iijima K. Metabolic syndrome, sarcopenia and role of sex and age: cross-sectional analysis of Kashiwa Cohort Study. PLoS ONE. 2014;9(11):e112718. http://dx.doi.org/10.1371/journal.pone.0112718

8. Yaffe K, Hann MN, Blackwell T, Cherkasowa E, Whitmer RA, West N. Metabolic syndrome and cognitive decline in elderly latinos: fidings from the Sacramento Area Latino Study of Aging study. JAGS. 2007;55:758-62. http://dx.doi.org/10.1111/j.1532-5415.2007.01139.x

9. Raffaitin C, Feart C, Le Goff M, Amieva H, Helmer C, Akbaraly TN, Gin H, Barberger-Gateau P. Metabolic syndrome and cognitive decline in French elders: the Three-City Study. Neurology. 2011;76:518-25. http://dx.doi.org/10.1212/WNL.0b013e31820b7656

10. Pereira AMVB. Prevalência de síndrome metabólica em idosos atendidos em um ambulatório de clínica médica de hospital universitário do Sul do Brasil e associação com declínio cognitivo. [dissertation]. [Porto Alegre]: Pontifícia Universidade Católica do Rio Grande do Sul; 2011. 166p.

11. Chaves ML, Camozzato AL, Godinho C, Piazenski I, Kaye J. Incidence of Mild Cognitive Impairment and Alzheimer Disease in Southern Brazil. J Geriatr Psych Neur. 2009;22:181-7. http://dx.doi.org/10.1177/0891988709332942

12. Rigo JC, Vieira JL, Dalacorte RR, Reichert CL. Prevalence of Metabolic Syndrome in a elderly community: comparision between three diagnostic methods. Arq Bras Cardiol. 2009;93:80-6.

13. Sociedade Brasileira de Cardiologia / Sociedade Brasileira de Hipertensão / Sociedade Brasileira de Nefrologia. VI Diretrizes Brasileiras de Hipertensão. Arq Bras Cardiol. 2010; 95(supl.1):1-51.

14. Freitas EV, Miranda RD. Parâmetros clínicos do envelhecimento e avaliação geriátrica ampla. In: Elizabete VF. Tratado de Geriatria e Gerontologia. 2a ed. Rio de Janeiro: Guanabara Koogan; 2006. p. 900-9.

15. Everitt BS, Dunn G: Applied multivariate data analysis. London: Edward Arnold; 1991.

16. Scuteri A, Najjar SS, Morrell CH, Lakatta EG. The metabolic syndrome in older individuals: prevalence and prediction of cardiovascular events: the Cardiovascular Health Study. Diabetes Care. 2005;28:882-7. http://dx.doi.org/10.2337/diacare.28.4.882

17. Ford ES, Giles WD, Dietz WH. Prevalence of the metabolic syndrome among US adults: Third National Health and Nutrition Examination Survey. JAMA. 2002;287:356-9. http://dx.doi.org/10.1001/jama.287.3.356

18. Mozaffarian D, Kamineni A, Prineas RJ, Siscovick DS. Metabolic syndrome and mortality in older adults: the Cardiovascular Health Study. Arch Intern Med. 2008;168:969-78. http://dx.doi.org/10.1001/archinte.168.9.969

19. Thomas F, Pannier B, Beneton A, Vischer UM. The impact of the metabolic syndrome - but not of hypertension - disappears in the elderly J Hypertens. 2011;29:663-8. http://dx.doi.org/10.1097/HJH.0b013e32834320dc

20. Ravaglia G, Forti P, Maioli F, Bastagli L, Chiapelli M, Montesi F, Bolondi L, Patterson C. Metabolic syndrome: prevalence and prediction of mortality in elderly individuals. Diabetes Care. 2006;29:2471-6. http://dx.doi.org/10.2337/dc06-0282

21. Simons LA, Simons J, Friedlander Y, J McCallum. Does a diagnosis of the metabolic syndrome provide additional prediction of cardiovascular disease and total mortality in the elderly? The Duboo Study. MJA. 2007;186:400-3.

22. He Y, Jiang Bin, Wang J, Feng K, Chang Q, Fan L, Li X, Hu FB. Prevalence of the metabolic syndrome and its relation to cardiovascular disease in an elderly chinese population. J Am Coll Cardiol. 2006;47:1588-94. http://dx.doi.org/10.1016/j.jacc.2005.11.074

23. Li R, Li W, Lun Z, Zhang H, Sun Z, Kanu JS, Qiu S, Cheng Y, Liu Y. Prevalence of metabolic syndrome in mainland China: a meta-analysis of published studies. BMC Public Health 2016;16:e296. http://dx.doi.org/10.1186/s12889-016-2870-y

24. Lopes MJC. Prevalência da síndrome metabólica no Brasil: um estudo de revisão. [monografia]. [Maringá]. Universidade Estadual de Maringá, 2009. 66 p.

25. Cabrera MAS, Gebara OCE, Diament J, Nussbacher A, Rosano G, Wajngarten M. Metabolic syndrome, abdominal obesity, and cardiovascular risk in elderly women. Int J Cardiol. 2007;114:224-9. http://dx.doi.org/10.1016/j.ijcard.2006.01.019

26. Roriz-Cruz M, Rosset I, Wada T, Sakagami T, Ishine M, Roriz-Filho JS, Cruz TRS, Rodrigues RP, Resmini I, Sudoh S, Wakatsuki Y, Nakagawa M, Souza AC, Kita T, Matsubayashi K. Stroke-independent association between metabolic syndrome and functional dependence, depression, and low quality of life in elderly community-dwelling brazilian people. JAGS. 2007;55:374-82. http://dx.doi.org/10.1111/j.15325415.2007.01068.x

27. Vieira CE, Peixoto MRG, Silveira EA. Prevalência e fatores associados à síndrome metabólica em idosos usuários do Sistema Único de Saúde. Rev Bras Epidemiol. 2014;17:805-17. http://dx.doi.org/10.1590/1809-4503201400040001

28. Wachholz PA, Masuda PY. Caracterização e prevalência de síndrome metabólica em idosos segundo dois critérios diagnósticos diferentes. Est Interdisc Envelhec. 2009;14:95-106. 
29. Paula HAA, Ribeiro RCl, Rosado LEPL, Pereira RSF, Franceschini SCC. Comparação de diferentes critérios de definição para diagnóstico de síndrome metabólica em idosas. Arq Bras Cardiol. 2010;95:346-53. http://dx.doi.org/10.1590/S0066-782X2010005000100

30. Saad MAN, Cardoso GP, Martins WA, Velarde LGC, Cruz Filho RA. Prevalência de síndrome metabólica em idosos e concordância entre quatro critérios diagnósticos. Arq Bras Cardiol. 2014;102:263-9.

31. Rosa CB, Agostini JA, Bianchi PD, Garces SBB, Hansen D, Moreira PR, Schwanke CHA. Síndrome metabólica e estado nutricional de idosos cadastrados no HiperDia. Sci Med. 2016;26(3):ID23100. http://dx.doi.org/10.15448/1980-6108.2016.3.23100

32. Aronow WS, Fleg JL, Pepine CJ, Artinian NT, Bakris G, Brown AS, Ferdinand KC, Ann Forciea M, Frishman WH, Jaigobin C, Kostis JB, Mancia G, Oparil S, Ortiz E, Reisin E, Rich MW, Schocken DD, Weber MA, Wesley DJ. ACCF/AHA 2011 Expert Consensus Document on Hypertension in the Elderly: A Report of the American College of Cardiology Foundation Task Force on Clinical Expert Consensus Documents Developed in Collaboration With the American Academy of Neurology, American Geriatrics Society, American Society for Preventive Cardiology, American Society of Hypertension, American Society of Nephrology, Association of Black Cardiologists, and European Society of Hypertension. J Am Coll Cardiol. 2011;57:2037-114. http://dx.doi.org/10.1016/j.jacc.2011.01.008

33. Benetos A, Bulpitt CJ, Petrovic M, Ungar A, Rosei AE, Cherubini A, Redon J, Grodzicki T, Dominiczak A, Strandberg T, Mancia G An Expert Opinion From the European Society of Hypertension-European Union Geriatric Medicine Society Working Group on the Management of Hypertension in Very Old. Hypertension. 2016;67:820-5. http://dx.doi.org/10.1161/HYPERTENSIONAHA.115.07020

34. Da Cruz IBM, Schwanke CHA. Reflexões sobre biogerontologia como uma ciência generalista, integrativa e interativa. Estud Interdiscipl Envelhec. 2001;3:7-36.

35. Rosemberg LS. Determinação dos pontos de corte de parâmetros antropométricos de obesidade e obesidade central para síndrome metabólica e risco cardiovascular em idosos da atenção básica. [Dissertação de Mestrado]. Rio Grande do Sul: Instituto de Geriatria e Gerontologia da Pontifícia Universidade Católica do Rio Grande do Sul; 2013. 113p.

36. Rojas R, Aguilar-Salinas CA, Jiménez-Corona A, Shamah-Levy T, Rauda J, Ávila-Burgos L, Villalpando S, Ponce EL. Metabolic syndrome in mexican adults. Results from the National Health and Nutrition Survey 2006. Salud Pública Méx. 2010;52:S11-18. http://dx.doi. org/10.1590/S0036-36342010000700004

37. Miccoli R, Bianchi C, Odoguardi L, Penno G, Pucci L, Del Prato S. Prevalence of the metabolic syndrome among italian adults according to ATP III definition. Nutr Metab Cardiovasc Dis. 2005 Aug;15:250-4. http://dx.doi.org/10.1016/j.numecd.2004.09.002

38. Sidorenkov O, Nilssen O, Brenn T, Martiushov S, Arkhipovsky VL, Grjibovski AM. Prevalence of the metabolic syndrome and its components in Northwest Russia: the Arkhangelsk study. BMC Public Health. 2010;10:23. http://dx.doi.org/10.1186/1471-2458-10-23

39. Devers MC, Campbell S, Simmons D. Influence of age on the prevalence and components of the metabolic syndrome and the association with cardiovascular disease. BMJ Open Diabetes Res Care. 2016;4:e00195. http://dx.doi.org/10.1136/bmjdrc-2016-000195

40. Moebus S, Hanisch JU, Aidelsburger P, Bramlage P, Wasem J, Jöckel K-H. Impact of 4 different definitions used for the assessment of the prevalence of the metabolic syndrome in primary healthcare: The German Metabolic and Cardiovascular Risk Project (GEMCAS). Cardiovasc Diabetol. 2007;6:22-32. http://dx.doi.org/10.1186/1475-2840-6-22

41. Chuang TJ, Huang CL, Lee CH, Hsieh CH, Hung YJ, Hung CF, Liang YJ, Chen YL, Hsia TL, Pei D. The differences of metabolic syndrome in elderly subgroups: A special focus on young-old, old-old and oldest old. Arch Gerontol Geriatr. 2016;65:92-7. http://dx.doi. org/10.1016/j.archger.2016.03.008

42. Perls T, Terry D. Understanding the determinants of exceptional longevity. Ann Int Med. 2003;139:445-9. http://dx.doi.org/10.7326/00034819-139-5 Part 2-200309021-00013

43. Frisard MI, Rood JC, Fang X, Su J, Welsh DA, Jazwinski SM, Ravussin E. Metabolic syndrome and risk factors for cardiovascular disease: are nonagenarians protected? Age. 2009;31:67-75. http://dx.doi.org/10.1007/s11357-008-9082-z

44. Curtis LH, Hammil BG, Bethel MA, Anstrom KJ, Gottdiener JS, Schulman KA. Costs of the metabolic syndrome in elderly individuals. Diabetes Care. 2007;30:2553-8. http://dx.doi.org/10.2337/dc07-0460

45. Laudisio A, Bandinelli S, Gemma A, Ferrucci L, Incalzi RA. Metabolic syndrome and functional ability in older age: The InCHIANTI study. Clin Nutr. 2014;33:626-33. http://dx.doi.org/10.1016/j.clnu.2013.08.005

46. Botoseneanu A, Ambrosius WT, Beavers DP, de Rekeneire N, Anton S, Church T, Folta SC, Goodpaster BH, King AC, Nicklas BJ, Spring B, Wang X, Gill TM. Prevalence of metabolic syndrome and its association with physical capacity, disability, and self-rated health in Lifestyle Interventions and Independence for Elders Study participants. JAGS. 2015;63:222-32. http://dx.doi.org/10.1111/jgs.13205

47. Penninx BW, Nicklas BJ, Newman AB, Harris TB, Goodpaster BH, Satterfield S, de Rekeneire N, Yaffe K, Pahor M, . Kritchevsky SB. Metabolic syndrome and physical decline in older persons: results from the Health, Aging and Body Composition Study. J Gerontol A Biol Sci Med Sci. 2009;64:96-102. http://dx.doi.org/10.1093/gerona/gln005

48. Blaum CS, West NA, Haan MN. Is the metabolic syndrome, with or without diabetes, associated with progressive disability in older mexican americans? J Gerontol A Biol Sci Med Sci. 2007;62:766-73. http://dx.doi.org/10.1093/gerona/62.7.766

49. Gottlieb MGV, Closs VE, Rosemberg LS, Borges CA, Baptista RR, Tavares GMS, Gomes I, Schneider, Schwanke CHA. Lack of association of body composition and functionality variables with metabolic syndrome in the elderly. Met Syndr Relat Disord. 2014;12:397-401. http:// dx.doi.org/10.1089/met.2014.0012

50. Liaw, FY. Kao TW, Wu LW, Wang CC, Yang HF, Peng TC, Sun YS, Chang YW, Chen WL. Components of metabolic syndrome and the risk of disability among the elderly population. Sci Rep. 2016;6:e22750. http://dx.doi.org/10.1038/srep22750

51. Wu TW, Chan HL, Hung CL, Lu IJ, Wang SD, Wang SW, Wu IJ, Wang LY, Yeh HI, Wei YH. (2014). Differential patterns of effects of age and sex on metabolic syndrome in Taiwan: implication for the inadequate internal consistency of the current criteria. Diabetes Res Clin Pract. 2014;105:239-44. http://dx.doi.org/10.1016/j.diabres.2014.04.027 